\title{
Accuracy of Triphasic Computerized Tomography in the Evaluation of Hemodynamics of Cirrhotic Liver
}

\author{
Mohamed R. Habaa', Mohamed M. Abdo ${ }^{2 *}$, Ahmed T. Ahmed', \\ Sara S. Souror ${ }^{1}$ \\ 'Department of Diagnostic Radiology, ${ }^{2}$ Department of Internal Medicine, Faculty of Medicine, Suez Canal \\ University, Egypt
}

\begin{abstract}
Background: Liver cirrhosis causes portal hypertension due to mainly parenchymal necrosis, then connective tissue deposition, nodular regeneration of hepatocyte, resulting in distortion of the hepatic architecture. In advanced cirrhosis the hepatic artery becomes engorged and tortuous. Spiral CT is used during the hepatic artery phase to identify and differentiate liver tumors and liver cirrhosis by detecting hemodynamic changes. CT can also display signs of fibrosis including abnormal morphology as left lobe hypertrophy, nodular edge, and regenerating nodules that appear low density in CT. If cirrhosis can be detected and graded by a non-invasive method this will be practically very helpful. Aim: this study aims to evaluate the severity of liver cirrhosis using triphasic CT by measuring hepatic tissue blood flow to improve the accuracy of diagnosis and staging of cirrhosis and subsequent management and follow-up and to avoid the more invasive liver biopsy. Subjects and Methods: A case control study was done and included patients attending the internal medicine and/or the radiology departments in Suez Canal university hospital. Results: 60 patients were included in this study 30 patients with previous history of liver disease compared to similar number of patients with no history of liver disease. There was statistically significant difference between region of interest (ROI) measurements in both groups and the difference was more observed between control group and Child-Pugh C patients. Conclusion: the study concluded that the ROI measurement of liver attenuation in Triphasic CT can be used to quantify liver cirrhosis as promising technique, and aid in the staging of hepatic cirrhosis.
\end{abstract}

Key Words: cirrhosis, triphasic CT, liver hemodynamics

\section{Introduction}

Liver cirrhosis causes portal hypertension due to mainly parenchymal necrosis, then connective tissue deposition, nodular regeneration of hepatocyte, resulting in distortion of the hepatic architecture ${ }^{(1-4)}$. Liver cirrhosis causes distortion of the normal liver blood flow hemodynamics, causing decreased portal

*Corresponding Author: d_m.abdo2014@yahoo.com 
flow to the liver and increased arterial flow. In advanced cirrhosis, the hepatic artery becomes enlarged and tortuous ${ }^{(3-}$ 5). Liver cirrhosis causes abnormal permeability of the sinusoid causing abnormal arterioportal communications. In severe cirrhosis, portal vein thrombosis is frequently seen ${ }^{(6)}$. The liver blood supply consists of two parts making helical computed tomography (CT) a profoundly appropriate method for liver imaging. Spiral CT allows a solitary breath-hold image acquisition staying clear of motion artifacts $(7)$. Quick image acquisition of spiral CT makes evaluation of the liver at different phases possible. The arterial and portal circulations to the liver have several communications. If vascular compromise happens, changes in the blood flow volume and direction occurs in individual vessels. These abnormalities can be recognized with spiral $\mathrm{CT}$ as high attenuation area on arterial phase images and returns to normal on portal phase images meaning there is an increased arterial blood flow and arterioportal shunting(7). Perfusion abnormalities are caused by liver cirrhosis, portal vein obstruction, hepatic trauma, hepatic neoplasms, hereditary hemorrhagic telangiectasia (HHT), hepatic vein obstruction, inflammatory changes, steal phenomenon by hypervascular tumors, aberrant blood supply, hepatic parenchymal compression, and others ${ }^{(8-11)}$. The liver is predominantly supplied by portal vein (75\%) and supplemented by hepatic artery (25\%). In liver cirrhosis, deposition of collagen in the space of Disse causes increased resistance to incoming blood flow in the sinusoids. This is responsible for the decreased portal flow, compensated by an increase in arterial flow through the hepatic arterial buffer response ${ }^{(12-14)}$. The most common noninvasive methods that show the liver morphology and are used for diagnosing liver cirrhosis are $\mathrm{CT}$, ultrasonography (US), and MRI. Cross-sectional imaging evaluates the hemodynamics in the liver providing additional vital data in the diagnosis of diffuse liver diseases ${ }^{(15-17)}$. Many modalities have been used to asses liver cirrhosis including ultrasound, doppler, fibroscan and magnetic resonance imaging. Although fibroscan can detect and grade cirrhosis it is unhelpful in the presence of ascites and in obese subjects.

\section{Subjects and Methods}

Type of the study: Case-control study. Study population: patients attending Hepatology and Radiology department in Suez Canal University hospital. A) Inclusion criteria: Patients with clinically confirmed cirrhosis with or without portal hypertension by clinical history, laboratory, ultrasound, and were definitively diagnosed as liver cirrhosis. The severity of the cirrhosis is evaluated using the Child-Pugh classification as shown below. Scoring system to evaluate chronic liver disease (CLD) severety. A (score 5-6), B (score 7-9) and $C$ (score 10-15). The inclusion criteria for the control group were as follows: normal liver function and hemodynamics, no prior liver disease (such as HBV or $\mathrm{HCV}$ ), and no evidence of portal vein thrombosis and decided to have to have triphasic CT study of whatever indication other than liver disease. B) Exclusion criteria: Patients with known contrast reaction. Creatinine clearance less than $30 \mathrm{ml} / \mathrm{min} / 1.73 \mathrm{~m}^{2}$. Pregnant wom- 
en. Patients in the pediatric age group (<18 years).

Imaging technique: All the studied patients were subjected to Triphasic CT of the liver. This was performed on a 16-slice CT scanner Toshiba Activion. Patients were kept fasting for at least 6 hours. Intravenous access (with $18 \mathrm{G}$ cannula) through antecubital vein. A CT scan of the liver was obtained without contrast medium during a breath hold at the end of expiration. The scan coverage was from the diaphragmatic dome to the lower poles of both kidneys ${ }^{(6)}$. CT examination of the total liver volume was acquired after intravenous injection of non-ionic contrast Optiray (loversol): $1.5 \mathrm{ml} / \mathrm{Kg}$ with overall dose from 80-100 $\mathrm{ml}$ at $3 \mathrm{ml} / \mathrm{s}$ using a automatic injector. Liver was scanned in arterial phase (scanning delay, 20-25 seconds), and portal phase (scanning delay, 45-60 seconds). circular region of interest (ROI) of approximately $200 \mathrm{~mm}^{2}$ was placed on four areas of the liver parenchyma: right posterior, right anterior, left medial, and left lateral segments, with caution avoiding the large vessels in the HAP and PVP, to measure the attenuation of the area selected $(2,17)$.

Severity of the cirrhosis is evaluated using the Child-Pugh classification

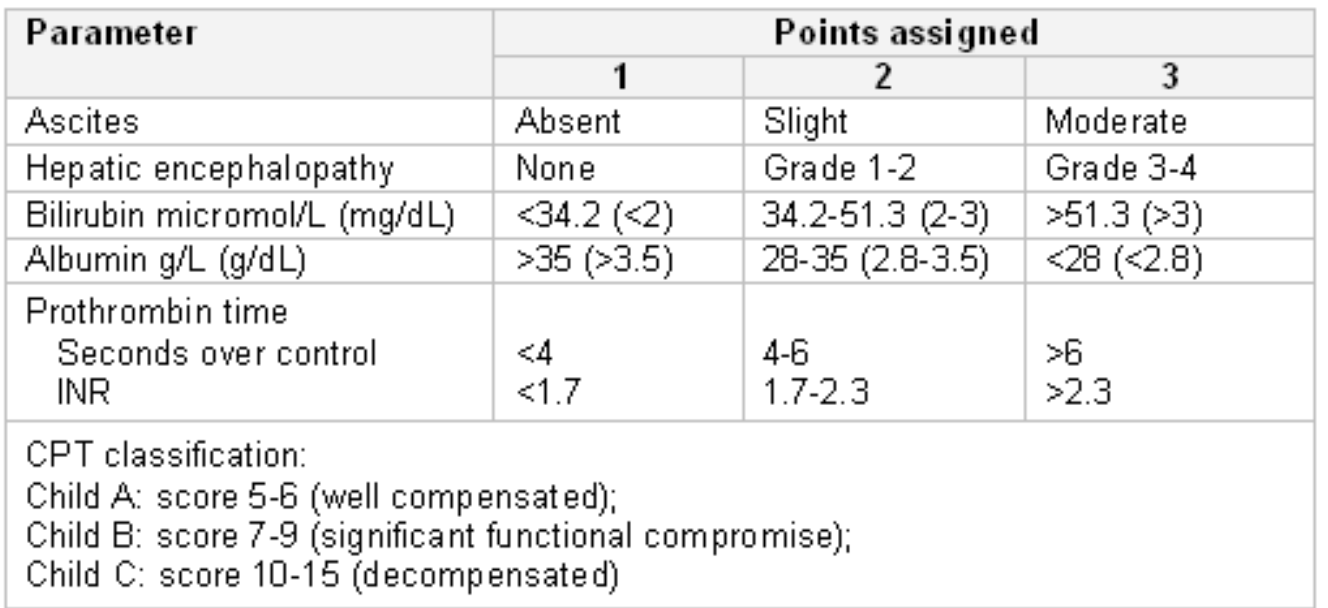

\section{Results}

The distribution of studied groups according to personal data is demonstrated in table (1). Of the 30 patients in the study group with CLD 20 patients had ascites and 10 patients did not. And only 7 patients had hepatic encephalopathy out of the 30 chronic liver disease patients (Table 2). All the 30 subjects in the study group were Hepatitis $C$ virus posi- tive, while in the control group were free (Table 3). The total subjects in our study were divided according to lab results and clinical data into a control group 30 subjects $(50 \%)$ and a study group 30 subjects (50\%). The 30 subjects in the study group were subdivided in our study according to lab results and clinical data into child Pugh class A 10 subjects (33.3\%), child Pugh class B 11 subjects (36.7\%), and child Pugh class C 9 
subjects (30.0\%), (Table 4). Average ROI measurement of all hepatic segments was calculated as a single measure and showed: The ROI measurement in
Hounsfield Units (HU) of the hepatic parenchyma in the portal phase was higher than the hepatic arterial phase in both study and control groups.

Table 1: Distribution of studied groups according to Personal data

\begin{tabular}{|c|c|c|c|c|c|c|c|}
\hline & \multicolumn{2}{|c|}{ Control Group $(n=30)$} & \multicolumn{2}{|c|}{ Study Group $(n=30)$} & \multirow{2}{*}{ p-value } \\
\hline & & & Freq. & $\%$ & Freq. & $\%$ & \\
\hline \multirow{4}{*}{ 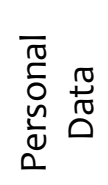 } & \multirow{2}{*}{ Gender } & Male & 19 & $63.3 \%$ & 18 & $60.0 \%$ & \multirow{2}{*}{0.12} \\
\hline & & Female & 11 & $36.7 \%$ & 12 & $40.0 \%$ & \\
\hline & Aore (vears) & Mean \pm SD & \multicolumn{2}{|c|}{$59.3 \pm 7.26$} & \multicolumn{2}{|c|}{$59.7 \pm 9.3$} & \multirow{2}{*}{0.25} \\
\hline & Age (years) & Range & \multicolumn{2}{|c|}{$50-82$} & \multicolumn{2}{|c|}{$28-75$} & \\
\hline
\end{tabular}

No statistically significant difference between two groups

Table 2: Distribution of studied groups according to history of clinical data

\begin{tabular}{|c|c|c|c|c|c|}
\hline & \multicolumn{2}{|c|}{$\begin{array}{l}\text { Control Group } \\
(n=30)\end{array}$} & \multicolumn{2}{|c|}{$\begin{array}{l}\text { Study Group } \\
\qquad(n=30)\end{array}$} \\
\hline & & Freq. & $\%$ & Freq. & $\%$ \\
\hline \multirow{6}{*}{ 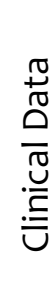 } & \multirow{2}{*}{ CLD } & 0 & $0.0 \%$ & 30 & $100.0 \%$ \\
\hline & & 30 & $100.0 \%$ & 0 & $0.0 \%$ \\
\hline & \multirow{2}{*}{ Ascites } & 0 & $0.0 \%$ & 20 & $66.7 \%$ \\
\hline & & 30 & $100.0 \%$ & 10 & $33.3 \%$ \\
\hline & \multirow{2}{*}{$\begin{array}{l}\text { Hepatic en- } \\
\text { cephalopathy }\end{array}$} & 0 & $0.0 \%$ & 7 & $23.3 \%$ \\
\hline & & 30 & $100.0 \%$ & 23 & $76.7 \%$ \\
\hline
\end{tabular}

Table 3: Distribution of studied groups according to liver infections

\begin{tabular}{|c|c|c|c|c|c|c|}
\hline & \multicolumn{2}{|c|}{$\begin{array}{c}\text { Control Group } \\
(n=30)\end{array}$} & \multicolumn{2}{|c|}{$\begin{array}{c}\text { Study Group } \\
(\mathrm{n}=30)\end{array}$} \\
\hline & & & Freq. & $\%$ & Freq. & $\%$ \\
\hline \multirow{6}{*}{ 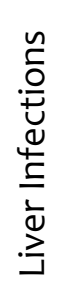 } & \multirow{2}{*}{ Hepatitis B } & Positive & 0 & $0.0 \%$ & 0 & $0.0 \%$ \\
\hline & & Negative & 30 & $100.0 \%$ & 30 & $100.0 \%$ \\
\hline & \multirow{2}{*}{ Hepatitis C } & Positive & 0 & $0.0 \%$ & 30 & $100.0 \%$ \\
\hline & & Negative & 30 & $100.0 \%$ & 0 & $0.0 \%$ \\
\hline & \multirow{2}{*}{$\begin{array}{l}\text { History of Schis- } \\
\text { tosomiasis }\end{array}$} & Yes & 0 & $0.0 \%$ & 0 & $0.0 \%$ \\
\hline & & No & 30 & $100.0 \%$ & 30 & $100.0 \%$ \\
\hline
\end{tabular}

Table 4: Distribution of study groups according to Child-Pugh classification

\begin{tabular}{|c|c|c|c|c|c|}
\hline \multicolumn{2}{|c|}{} & \multicolumn{2}{c|}{ Control Group $(\mathrm{n}=30)$} & \multicolumn{2}{c|}{ Study Group $(\mathrm{n}=30)$} \\
\cline { 3 - 6 } \multicolumn{2}{c|}{} & Freq. & $\%$ & Freq. & $\%$ \\
\hline \multirow{3}{*}{$‡$} & Control & 30 & $100.0 \%$ & 0 & $0.0 \%$ \\
\cline { 2 - 6 } & Child-Pugh A & - & - & 10 & $33.3 \%$ \\
\cline { 2 - 6 } & Child-Pugh B & - & - & 11 & $36.7 \%$ \\
\cline { 2 - 6 } & Child-Pugh C & - & - & 9 & $30.0 \%$ \\
\hline
\end{tabular}


The ROI measurement in $\mathrm{HU}$ of the hepatic parenchyma in the hepatic arterial phase showed no significant difference as compared to the control group. However, the HU in the portal phase of the study group was significantly lower than that the portal venous phase in the control group. There was statistical difference between $\mathrm{HU}$ in the control group and child Pugh B, A and B, A and $C, B$ and $C$. There was a significant difference between the control group and child Pugh C, but non between the control and child Pugh A (Table 5). In the portal phase, ROI measurement of the control group was slightly higher than the study group. However, there was almost no difference between the control group and Child-Pugh grade A, but there was a significant difference between the control and Child-Pugh $C$ as well as Child-Pugh $A$ and $C$. there was a decrease in ROI measurement with increasing Child-Pugh grades" (Table 6).

\section{Discussion}

Liver cirrhosis is the outcome for CLD. It has an increasing prevalence and is the seventh leading cause of death. Approximately 25,000 die yearly from its complications. Hepatitis C virus (HCV) infection is hyper-endemic in Egypt, where schistosomiasis is common. Chronic HCV is the main cause of liver cirrhosis and cancer in Egypt. The rate of HCV seroprevalence exceeds $30 \%$ in the subgroup of patients aged 40 to 67 years ${ }^{(11)}$.

Table 5: ROI (region of interest) measurement in Hounsfield unit (HU) of different groups measured in ALL Liver segments at each phase

\begin{tabular}{|c|c|c|c|c|c|c|}
\hline & \multicolumn{4}{|c|}{ ROI measurement- All segments } \\
\hline & & & Control Group & Child-Pugh A & Child-Pugh B & Child-Pugh C \\
\hline \multirow{6}{*}{ 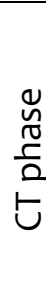 } & \multirow{2}{*}{$\begin{array}{c}\text { Pre- } \\
\text { contrast }\end{array}$} & Mean \pm SD & $49.6 \pm 4.3$ & $45.5 \pm 13.4$ & $45.9 \pm 5.2$ & $44.5 \pm 6.3$ \\
\hline & & Range & $35-61$ & $11-72$ & $36-56$ & $31-55$ \\
\hline & \multirow{2}{*}{$\begin{array}{l}\text { Arterial } \\
\text { phase }\end{array}$} & Mean \pm SD & $57.8 \pm 4.9$ & $49.1 \pm 9.6$ & $52.9 \pm 7.5$ & $48.9 \pm 8.7^{*}$ \\
\hline & & Range & $44-70$ & $22-62$ & $40-74$ & $26-64$ \\
\hline & \multirow{2}{*}{$\begin{array}{l}\text { Portal } \\
\text { phase }\end{array}$} & Mean \pm SD & $77.7 \pm 10.7$ & $75.0 \pm 12.4$ & $67.1 \pm 10.6^{*}$ & $57.2 \pm 8.1^{*}$ \\
\hline & & Range & $55-115$ & $50-105$ & $51-94$ & $41-74$ \\
\hline
\end{tabular}

*Significant difference vs. control group.

Table 6: ROI (region of interest) measurement in Hounsfield unit (HU) of different groups in PORTAL PHASE.

\begin{tabular}{|c|c|c|c|c|c|}
\hline \multicolumn{2}{|c|}{} & \multicolumn{4}{|c|}{ ROI measurement- Portal phase } \\
\cline { 3 - 6 } \multicolumn{2}{|c|}{} & $\begin{array}{c}\text { Rt post. seg- } \\
\text { ment }\end{array}$ & Rt ant. segment & $\begin{array}{c}\text { Lt med. Seg- } \\
\text { ment }\end{array}$ & $\begin{array}{c}\text { Lt lat. Seg- } \\
\text { ment }\end{array}$ \\
\hline \multirow{3}{*}{\begin{tabular}{c}
\multirow{2}{*}{} \\
\multirow{3}{*}{}
\end{tabular}} & Control & $75.9 \pm 10.3$ & $78.2 \pm 11.1$ & $79.2 \pm 9.8$ & $77.6 \pm 11.8$ \\
\cline { 2 - 6 } & Child-Pugh A & $75.9 \pm 13.8$ & $72.5 \pm 12.5$ & $75.3 \pm 12.3$ & $76.4 \pm 12.7$ \\
\cline { 2 - 6 } & Child-Pugh B & $66.5 \pm 11.4$ & $66.2 \pm 9.6^{*}$ & $67.8 \pm 11.1^{*}$ & $67.8 \pm 11.8^{*}$ \\
\cline { 2 - 6 } & Child-Pugh C & $58.6 \pm 7.8^{*}$ & $51.4 \pm 6.8^{*}$ & $60.1 \pm 9.1^{*}$ & $58.7 \pm 6.6^{*}$ \\
\hline
\end{tabular}

*Significant difference vs. control group. 
Case 1-(control)

Male patient, 40 years old, with a known liver hemangioma for follow up but otherwise no chronic liver disease.

\begin{tabular}{|l|c|c|}
\hline Laboratory tests & Reference range & Patient \\
\hline Bilirubin T $(\mathrm{mg} / \mathrm{dL})$ & $0.1-1.2$ & 0.4 \\
\hline S. albumin $(\mathrm{g} / \mathrm{L})$ & $3.5-5$ & 4.7 \\
\hline PT (sec prolonged) & $9.5-13.8$ seconds (None) & 12 \\
\hline
\end{tabular}

Triphasic CT ROI in HU of different liver segments in different CT phases.

\begin{tabular}{|l|c|c|c|c|}
\hline \multirow{2}{*}{} & \multicolumn{4}{|c|}{ Regions } \\
\cline { 2 - 5 } & Rt post. & Rt ant. & Lt med. & Lt lat. \\
\hline Pre-contrast & 35 & 40 & 42 & 43 \\
\hline Arterial phase & 46 & 49 & 53 & 44 \\
\hline Portal phase & 80 & 82 & 81 & 82 \\
\hline
\end{tabular}

Case 2 (Child pugh grade A)

Female patient, 44 years old, complaining of abdominal pain and weight loss with a history of chronic liver disease. Suspected crohn's disease.

\begin{tabular}{|c|c|c|c|c|}
\hline Laboratory tests & & & & \\
\hline Point score & 1 & 2 & 3 & Patient \\
\hline Total bilirubin (mg/dL) & $<2$ & $2-3$ & $>3$ & 1.8 \\
\hline Serum albumin (g/L) & $>3.5$ & $2.8-3.5$ & $<2.8$ & 3 \\
\hline PT (sec prolonged) & $<4$ & $4-6$ & $>6$ & 2 \\
\hline Ascites & None & Mild & Moderate to severe & None \\
\hline Hepatic encephalopathy & None & Grad I-II & Grade III-IV & None \\
\hline \multicolumn{5}{|l|}{ Child Pugh classification } \\
\hline Classification & A & $\mathrm{B}$ & C & subject \\
\hline Total points & $5-6$ & $7-9$ & $10-15$ & $6-A$ \\
\hline
\end{tabular}

\section{Triphasic CT ROI in HU of different liver segments in different CT phases.}

\begin{tabular}{|l|c|c|c|c|}
\hline & \multicolumn{4}{|c|}{ Regions } \\
\cline { 2 - 5 } & Rt post. & Rt ant. & Lt med. & Lt lat. \\
\hline Pre-contrast & 39 & 41 & 43 & 42 \\
\hline Arterial phase & 48 & 50 & 50 & 53 \\
\hline Portal phase & 66 & 65 & 69 & 77 \\
\hline
\end{tabular}

\section{Impression}

Increase in ROI measurement in the arterial and portal phases compared to the precontrast phase. The ROI measurement in the portal phase was significantly higher than arterial and pre-contrast phases indicating early liver disease with good liver perfusion and function.

All chronic liver disease patients have liver fibrosis that develops into cirrhosis.
About 20 to 30 percent of subjects without cirrhosis at presentation will 
develop its complications which are: hepatocellular carcinoma, hepatic failure, ascites, variceal bleeding, hepatic encephalopathy. Portal hypertension will eventually develop and has a high prevalence in Egypt. It causes development of portosystemic collaterals with compensatory portosystemic shunting and disturbed intrahepatic circulation $^{(14,19)}$. Many modalities have been used to asses liver cirrhosis including ultrasound, doppler, fibroscan and magnetic resonance imaging. Although fibroscan can detect and grade cirrhosis it is unhelpful in the presence of ascites and in obese subjects. Noninvasive methods to estimate liver cirrhosis are increasingly being used in clinical practice, including serum biomarkers, and imaging modalities. If noninvasive methods could asses liver cirrhosis, then further assessment with liver biopsy with its limitations may not be needed. Ultrasound and Computed tomography are non-invasive methods that can assess the morphologic abnormalities of $\operatorname{cirrhosis}^{(20)}$. The present study uses triphasic CT to detect the severity of hepatic cirrhosis using a noninvasive quantitative measurement of hepatic tissue blood flow to improve accuracy of diagnosis and staging of cirrhosis and subsequent management and follow-up. The main point of this study was to compare the hepatic parenchymal enhancement obtained in triphasic CT imaging of cirrhotic patients to that of the control group with normal liver function and to evaluate the possibility of estimating the liver hemodynamics of cirrhotic patients using ROI (region of interest) measurement in HU (Hounsfield Unit). The total subjects in our study were divided according to lab results and clinical data into a control group 30 subjects (50\%) and a study group 30 subjects (50\%). The 30 subjects in the study group were subdivided in our study according to lab results and clinical data into child Pugh class A 10 subjects (33.3\%), child Pugh class B 11 subjects (36.7\%), and child Pugh class C 9 subjects (30.0\%). There was statistically significant difference between ROI measurements in the control group and child-Pugh $B$, child-Pugh $A$ and $B$, ChildPugh $A$ and $C$, Child-Pugh $B$ and $C$. There was a significant difference between the control group and Child-Pugh $\mathrm{C}$, but non between control group and Child-Pugh A. In our study, there was a significant decrease in ROI measurement in the portal phase in Child-Pugh $C$ as compared to the control group in all liver segments due to the presence of portal hypertension and possible decrease in parenchymal portal venous flow resulting in decreased blood flow and consequently decreased blood perfusion and decreased contrast perfusion throughout the liver. Cirrhotic nodules or fibrotic scars obliterate hepatic vascular space and thus raising resistance to portal flow $^{(8)}$. Similarly, in our study, the ROI measurement in hepatic parenchyma was found to be almost similar in the arterial phase compared to the control group and lower in the portal phase for the study group than in the control group. For the different Child-Pugh grades, our study demonstrated that ROI measurement decreased as liver function (Child-Pugh grade) deteriorated in the study group, which may be a result of the increased arterial supply and decreased portal supply of the liver parenchyma in advanced liver cirrhosis ${ }^{(11)}$. ROI measurement could be a suc- 
cessful predictive indicator for liver hemodynamics of cirrhosis patients when it can be used as a quantitative method because it reflects changes in hemodynamics and correlates with Child-Pugh grade. Our study agrees with the opinion that the iodine content (contrast enhancement in our study) measured with spectral CT in the arterial phase is proportional to the arterial blood flow, and the porto-venous phase is mainly proportional to the whole liver blood flow $^{()}$. In our study, the ROI measurement of hepatic parenchyma was found to be higher in the portal phase and lower in the arterial phase for patients in the study group than in the control group, resulting in an elevated CEF for cirrhotic patients. This value was significantly lower than that for patients with normal liver functions.

\section{Conclusion}

We conclude that, the ROI measurement of liver attenuation in Triphasic CT can be used to quantify liver fibrosis as a promising technique, and aid in the staging of hepatic cirrhosis; with many advantages, which can be summarized as follows: Non-invasive technique. No anesthetic risk. Can be used if liver biopsy is contraindicated. quantify hemodynamic changes in liver cirrhosis

\section{References}

1. Baron $\mathrm{RL}$, Morimoto $\mathrm{M}$, Peterson MS (2001): Screening the cirrhotic liver for hepatocellular carcinoma with CT and MR imaging: opportunities and pitfalls. Radiographics 21, $P P: 117-132$.

2. Bataller R, Brenner DA (2005): liver fibrosis, The Journal of Clinical In- vestigation http://www.jci.org Volume 115 Number 2 February 2005; 115:209-218.

3. Bismuth H (1982): Anatomical surgery and surgical anatomy of the liver. World J Surg 6: 3-9.

4. Catheline S, Wu F, Fink M. (1999b): A solution to diffraction biases in sonoelasticity: The acoustic impulse technique. J Acoust Soc Am 1999b; 105:2941-2950.

5. Chatila R, Ferayorni L \& Groszmann RJ. Local arterial vasoconstriction induced by octreotide in patients with cirrhosis. Hepatology 2000; 31: 572-576.

6. Chen ML, Zeng QY, Huo JW, et al. Assessment of the hepatic microvascular changes in liver cirrhosis by perfusion computed tomography. World J Gastroenterol. 2009; 15:3532-3537

7. Chusilp CJ, Scott G, Peter R (2002): Hepatobiliary and pancreatic radiology, I st ed. New York: Thieme; PP122.2. D.S. Hugues and J.L.Kelly (1953):Second-order elastic deformation of solids , Phys .Rev. 92,11451149.

8. Cichoz-Lach H, Celiñski K, Slomka M, et al. Pathophysiology of Portal Hypertension. J Physiol Pharmacol 2008; 59 (2): 231.238.

9. Colombato LA, Albillos A \& Groszmann RJ. Temporal relationship of peripheral vasodilatation, plasma volume expansion and the hyperdynamic circulatory state in portal-hypertensive rats. Hepatology 1991; 15: 323-328.

10. Cui X, Friedrich-Rust M, De Molo C et al. Liver elastography, comments on EFSUMB elastography guidelines 2013. World J Gastroenterol. 2013; 14;19 (38): 6329-47.

11. Darwish $M A$, et al.: High seroprevalence of hepatitis $A, B, C$, and $E$ vi- 
ruses in residents in an Egyptian village in the Nile delta; 1996.

12. De Vries PJ, de Hooge P, Hoekstra JB, van Hattum J. Blunted postprandial reaction of portal venous flow in chronic liver disease, assessed with duplex Doppler: significance for prognosis. J Hepatol 1994; 21:966-973

13. DeLeve LD, Shulman HM, McDonald GB. Toxic injury to hepatic sinusoids: sinusoidal obstruction syndrome (veno-occlusive disease). Semin Liver Dis 2002; 22: 27-42.

14. Durand F, Valla D. Assessment of the prognosis of cirrhosis: ChildPugh versus MELD. Journal of Hepatology 42 (2005) S100-S107.

15. Elsayes KM, Narra VR, Mukundan G, Lewis Jr. JS, Menias CO, Heiken JP. $M R$ imaging of the spleen: spectrum of abnormalities. Radiographics, vol. 25, no. 4, pp. 967-982, 2005.

16. Foucher J, Chanteloup E, Vergniol J, Castéra L, Bail BL, Adhoute X, Bertet $J$, Couzigou $P$ and Lédinghen VD
(2005): (FibroScan): a prospective study Diagnosis of cirrhosis by transient elastography. Gut 2006; 55; 403-408.

17. Foley WD, Mallisee TA, Hohenwalter MD, Wilson CR, Quiroz FA, Taylor AJ. Multiphase hepatic $C T$ with a multirow detector $\mathrm{CT}$ scanner. AR Am J Roentgenol 2000;175: 679-85.24.

18. Franchis RD, Primignani M. Natural history of portal hypertension in patients with cirrhosis, Clinics in Liver Disease. 2001; 5 (3): 645-663.

19. Fried MW, Connaghan DG, Sharma S et al. Transjugular intrahepatic portosystemic shunt for the management of severe venoocclusive disease following bone marrow transplantation. Hepatology 1996; 24: 588-91.

20. Friedrich-Rust $M$, Ong $M$, Herrmann E, Dries V, Samaras P, Zeuzem S, Sarrazin C (2007): Real-Time Elastography for Noninvasive Assessment of Liver Fibrosis in Chronic Viral Hepatitis AJR 2007; 188:758764. 